\title{
VISUALISATION OF COMPLEX 3D CITY MODELS ON MOBILE WEBBROWSERS USING CLOUD-BASED IMAGE PROVISIONING
}

\author{
M. Christen* a , S. Nebiker ${ }^{a}$ \\ ${ }^{a}$ Institute of Geomatics Engineering, FHNW University of Applied Sciences and Arts Northwestern Switzerland - \\ (martin.christen, stephan.nebiker)@fhnw.ch
}

\author{
Commission III, WG 5
}

KEY WORDS: Big Data, Rendering Geospatial Data, Processing Geospatial Data, Image Based Provisioning, Standards

\begin{abstract}
:
Rendering large city models with high polygon count and a vast amount of textures at interactive frame rates is a rather difficult to impossible task as it highly depends on the client hardware, which is often insufficient, even if out-of-core rendering techniques and level of detail approaches are used. Rendering complex city models on mobile devices is even more challenging. An approach of rendering and caching very large city models in the cloud using ray-tracing based image provisioning is introduced. This allows rendering large scenes efficiently, including on mobile devices. With this approach, it is possible to render cities with nearly unlimited number of polygons and textures.
\end{abstract}

\section{INTRODUCTION AND RELATED WORK}

Over the last decade, we have witnessed the emergence and maturing of web-based 3D geoinformation services, namely 3D virtual globes. This has also spurred ongoing research both in the automated generation and in the efficient visualisation of very large and increasingly complex 3D city models. Some of the main trends in urban $3 \mathrm{D}$ reconstruction include procedural modelling approaches (e.g. Haegler et al., 2009) as well as image-based reconstruction - both from oblique aerial and ground-based imagery (Haala and Kada, 2010; Musialski et al., 2013). Both approaches are used to automatically reconstruct very large urban scenes with huge image textures and increasingly complex geometries and with potentially very large variations in level of detail. Over the last few years a great amount of research has been conducted on efficient web-based 3D graphics in general (Evans et al., 2014) and in web- and cloud-based architectures for large and complex geospatial 3D scenes in particular (Christen et al., 2012 \& 2014).

Recently some interesting approaches for rendering massive geospatial datasets on mobile devices have been introduced. Hildebrandt et al. (2011), for example, proposed an architecture for server-side rendering of massive 3D panoramas using cube maps. In their solution the $3 \mathrm{D}$ model is preprocessed and provided as a panorama image from the server. Another approach is the provisioning of massive, virtual 3D city models on web browsers, smartphones or tablets assembled from artificial oblique image tiles. It concluded that when using image provisioning techniques, the complexity of the 3D city model data is decoupled from data transfer complexity (Klimke et al., 2014). Also noteworthy is the HuMoRS system (Huge models Mobile Rendering System), where a networked 3D graphics system for interactively streaming and exploring massive 3D mesh models on mobile devices has been proposed (Rodríguez, 2014).

The Open Geospatial Consortium (OGC) and the Web3D Consortium have both been actively addressing the need for interoperability in the field of geospatial 3D visualisation, in particular handling complex datasets, mastering access speed, and allow a diversity of devices. The Web3D Consortium has focused on open standards for real-time 3D visualization, including streaming. The OGC has focused on developing a service interface to provide interoperable access to 3D geospatial data servers. In 2012, a group of OGC members, building on work done in both organizations, completed the 3D Portrayal Interoperability Experiment to develop and evaluate best practices for 3D portrayal services (Open Geospatial Consortium, 2015). The current state of the portrayal service has been used to implement the proposed $3 \mathrm{dmaps} . \mathrm{ch}$ framework.

In this paper we first introduce our cloud-based image provisioning approach including the underlying architecture. We then introduce two very demanding use cases with large and complex 3D city models, one of which was created using image-based modelling, the other one using procedural modelling. We subsequently present first results obtained with our cloud-based image provisioning prototype and provide an outlook and conclusion.

\section{PROPOSED CLOUD-BASED TILED IMAGE PROVISIONING APPROACH}

In the following, we introduce the $3 \mathrm{dmaps} . c h$ framework, which uses the view-extension of the $3 \mathrm{D}$ portrayal service for server and the browser-based visualisation. For the visualisation, an image-based provisioning approach is used. Different views of a city model can be rendered, cached in the cloud, and streamed to the client in the form of image tiles. The rendering step supports different zoom levels and view orientations of the city model. The resulting images follow the principle of G-buffers (Saito 1990). The first image tile type is a diffuse map, containing unlit colors of the scenery, the second tile type is a normal map containing normals encoded in RGB. With these two tile types, it is possible to support dynamic lighting of the scene. A third tile type contains ids of the $3 \mathrm{D}$ objects in the scene encoded as RGBA color values, which can be used for the

* Corresponding author 
identification of objects. The fourth image tile type is the Zbuffer tile containing the 32-bit per pixel depth values. With this tile type, it is possible to get the 3D position of every pixel on screen. All tiles are streamed as images in the png format since almost any kind of data can be encoded in and decoded from RGB and RGBA images (Christen, 2005).

\subsection{Creating New Scenes}

The 3dmaps.ch web application consists of several python scripts, which are handled by a web interface. The web interface uses flask (Flask, 2015; Lerner, 2014) and makes use of MongoDB, an open-source, document database designed for scalability (MongoDB, 2015). The web interface is optional and the scripts can also be called directly.

Scene creation - First, there is the script "createScene.py" which creates a new scene. A scene consists of assets such as 3D geometry, textures, and material definitions. If a 3D format without semantics is used, 3D geometries should be handled as a file per building so an id per Object can be created. In this case the id is simply the filename of the geometry.

Addition of scene contents - New geometry can be added to a scene by uploading the data via the web interface or by calling the script "addFilesToScene.py". If orthophotos are used in combination with elevation data, they must be provided as 3D geometry with textures. A python script to create a 3D model from orthophotos and elevation data has been developed for this purpose. Once the scene is complete it can be optimized by removing duplicate vertices, faces and material definitions.

As the subsequent rendering performance is critical, a spatial data structure is created for acceleration. The bounding volume hierarchy $(\mathrm{BVH})$ is of the most popular spatial data structures (Barringer 2014). The BVH is used for 3D objects, including all buildings and terrain. To support very large $3 \mathrm{D}$ cities and to keep the memory footprint low, the BVH is stored as multiple files in the file system. The textures are converted to a format with multiresolution support to reduce time for pixel lookup.

\subsection{Rendering Scenes}

Once all assets are uploaded to the server so called renderings can be configured. These rendering configurations consist of the $3 \mathrm{D}$ tile types to be rendered, the view directions which are used to render the view of the $3 \mathrm{D}$ city. The view position can be configured using a pitch angle (Figure 1) and a look-at angle (Figure 2). In Figure 2, we see a look-at angle of $0^{\circ}$ looking north and a second view with an angle of $45^{\circ}$ looking northwest. The pitch in this example is $22^{\circ}$. There is no limitation as to how many different renderings can be created. A new rendering is defined using the script "createRendering.py".

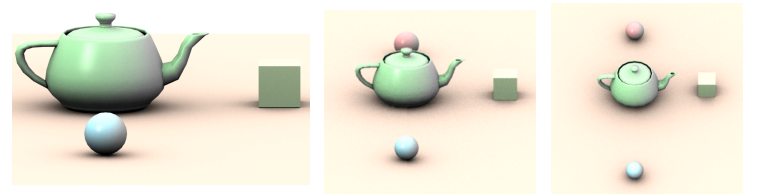

Figure 1: A scene rendered with a pitch of $10^{\circ}, 22.5^{\circ}$, and $45^{\circ}$

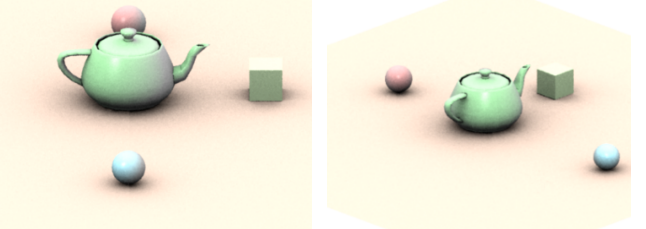

Figure 2: Using different look-at directions

Perspective image tiles are created in a quadtree structure, as shown in Figure 3. Instead of using a perspective projection for the oblique views, a parallel projection. As a result a uniform level of detail can be used across the entire field of view and that buildings are shown with a uniform scale across the entire screen. An additional advantage of the quadtree structure is that only the lowest pyramid level needs to be rendered and that the remaining levels can be calculated using image processing operations. Thus, the full potential of the acceleration structure is exploited. A simple tiling example is shown in Figure 4.

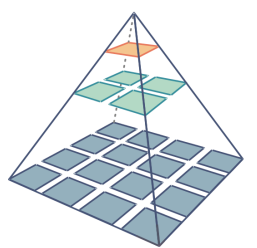

Figure 3: Quadtree scheme for 3D tiles

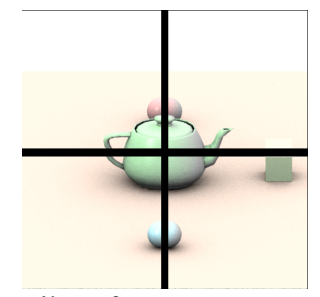

Figure 4: 3D tiles of a test scene at zoom level 1

The actual scene rendering is done using ray-tracing, to handle large amounts of textures and polygons in an efficient way. For the time being, an own implementation of a ray-tracer was not a priority, so RenderMan (Renderman 2015) is used for rendering the tiles. However, it would be possible to use other renderers instead or to implement our own rendering solution.

The tiles to be rendered are stored as render-jobs in MongoDB. The rendering can be distributed in the cloud using as many nodes as needed for processing tiles since each node processes a new job retrieved from the database. Once all full-resolution tiles are processed the remaining zoom levels are calculated and stored in the cloud.

\subsection{Server Architecture}

The server architecture follows the principles of the upcoming OGC 3D portrayal service. In the first version, the View Extension has been implemented for retrieving the images from the server. For each tile, a new view is calculated. The server implementation also supports a caching strategy, so that each request is stored and not being calculated twice. The architecture is shown in Figure 5. 


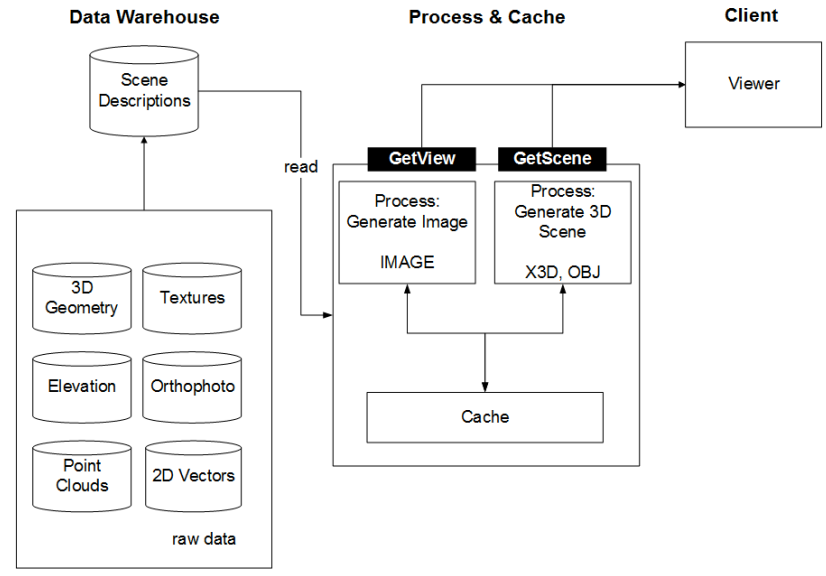

Figure 5: Client/Server Architecture of 3dmaps.ch

\subsection{Viewer API}

For displaying the scene in a webbrowser a JavaScript library has been implemented. It is called "map3d.js" and can be used to create custom scenes.

As shown in Listing 1, a new 3D map is created by specifying an id of a canvas element. Then a new layer is created. For the layer the URL(s) to the preprocessed tiles are provided. It is possible (and recommended) to specify many tile servers to speed up downloads, as the number of simultaneous persistent connections per server is limited in Web browsers. The layer is then added to the map. Currently, only a single 3D layer can be added to the map. In future, there will be support of multiple 3D layers, which can be combined using their z-buffers. Finally, we add two markers to the scene. The markers use real 3D positions of the objects. With this, the $3 \mathrm{D}$ scene is ready and the result is shown in Figure 6.

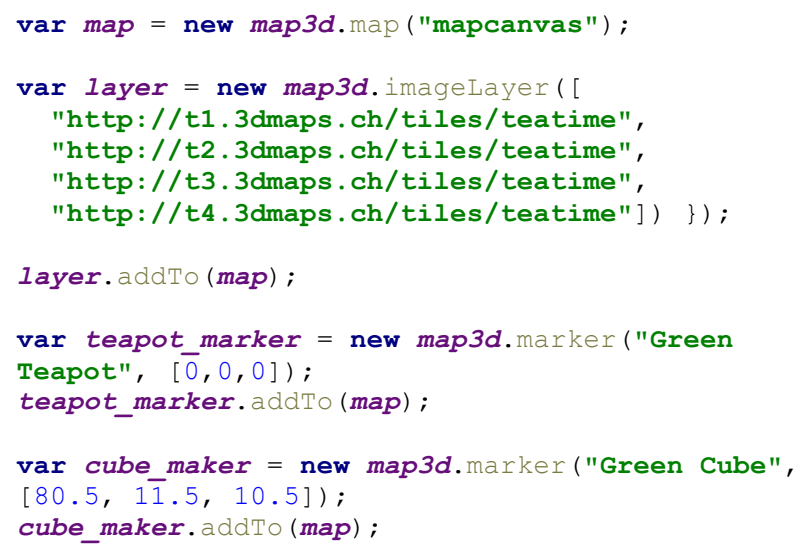

Listing 1. Using the viewer API

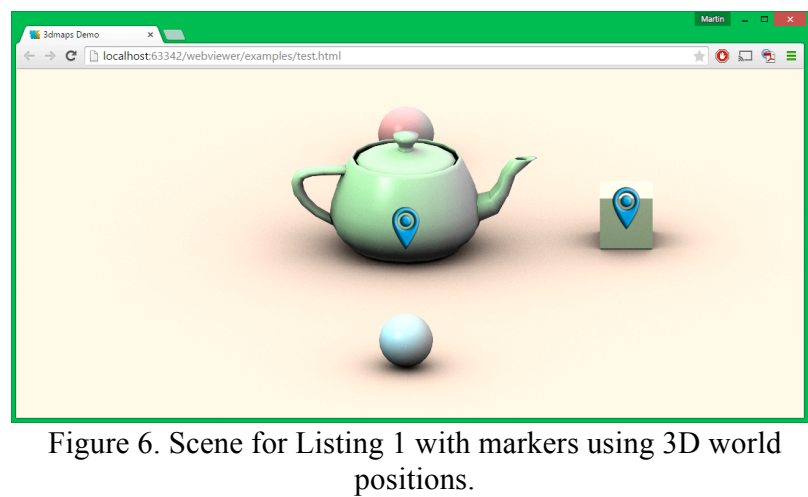

The map3d.js library contains an event system supporting custom events such as clicking on an object or building, selecting a marker, and so on. A building can be highlighted according to its id, which is retrieved from the color id tile. The highlighting is then calculated and displayed as shown in Figure 7.

The viewer supports both canvas-only and WebGL-based rendering modes. The WebGL version converts all tiles to textures. Certain operations like highlighting are done completly on the GPU. The texture of the color id tile and the RGB texture are combined in the fragment shader. Also the Z-buffer is written using the WebGL extension "EXT_Frag_Depth", which will also be part of WebGL 2.0. This allows the combination of polygonal 3D objects with the image-based view.

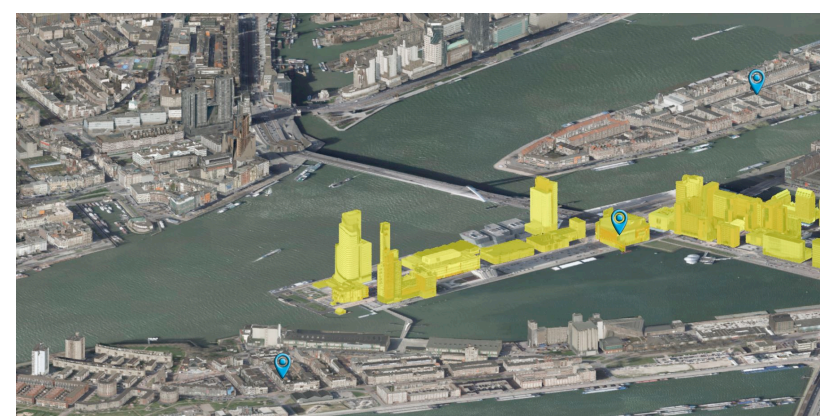

Figure 7. Highlighting objects

\section{USE CASES}

Currently there is a trend toward publishing textured 3D city models in the CityGML format as open data. These city models can be freely downloaded and usually be used for any purpose. This promotes the development of new algorithms and visualizations with real city data. Today, the list of city models released as open data includes: Lyon (France), Hamburg (Germany), Monréal (Canada), Rotterdam (Netherlands), and Berlin (Germany). For the first use case, we chose the modern city of Rotterdam, representing one of the open datasets. In our second case study, we use a procedural city model of the historic roman city of Augusta Raurica.

\subsection{Use Case 1: The Rotterdam Dataset}

The city model of Rotterdam is available to the public as CityGML dataset. The dataset consists of:

- 90 CityGML files with a total size of $2.72 \mathrm{~GB}$

- 26 '474 textures with a size of $1024 \times 1024$, an uncompressed total data volume of around $77 \mathrm{~GB}$ 
CityGML is an XML-based format for the storage and exchange of virtual 3D city models. It is therefore not a format optimized for viewing and can't simply be converted to an optimized viewing format. In addition, the dataset consists of texture atlases which are not optimized at all. Optimal results would be achieved if a $3 \mathrm{D}$ designer would manually redesign all buildings using 3D modelling software. However, we did not have the resources and the intention of doing so. Instead, we implemented a more general way for converting the models in an automated process. In this process we use all original textures and 3D data. In a first step the data is converted from CityGML to Wavefront OBJ format using FME (Safe Software). In a second step the materials and geometry are optimized using a python script. The script does:

- remove untextured faces, as we only want textured buildings

- remove triangles with double vertices

- optimize materials: use only one material per texture

- flip axis and face-orientation so we have a right handed coordinate system for further processing

The textures themselves are not changed as we did not want to reduce the given image quality. However, the original image quality is quite low as we can see when loading the converted dataset in a viewer such as MeshLab (Figure 8). Some city blocks however were too big to be loaded with all textures.

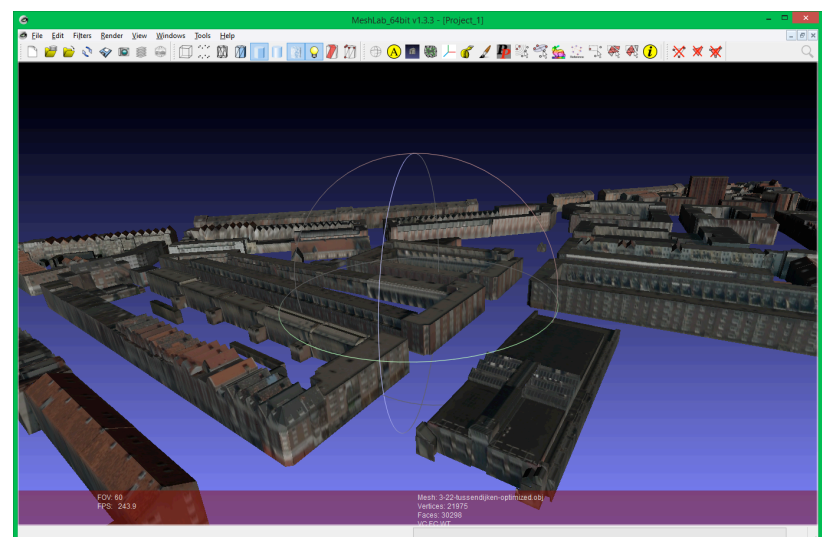

Figure 8. 3D Visualization of a single district containing 151 textures using MeshLab

In addition to the buildings, we used the orthophoto of Rotterdam, which is also available as open data (Figure 9). The file was downloaded from the Rotterdam open data portal in ECW Format. It has a size of $620^{\prime} 000 x$ 248'696 Pixels. The uncompressed size of the image is around $430 \mathrm{~GB}$. With the Wavelet based ECW compression the file is reduced to $8.4 \mathrm{~GB}$. For the 3D visualization we created 10 '000 tiles with a size of 4096x4096 Pixels. This was necessary, since the RenderMan renderer does not support ECW Format natively. The tiling has been implemented using a simple python script. As part of the tiling process, the script also creates a 3D file in the Wavefront OBJ Format, including the materials.

The 3D map of Rotterdam has been processed using 8 zoom levels on a single machine. For the demo a total of 87381 tiles have been preprocessed and stored in the cloud. A visual impression of the Rotterdam scene is shown in Figure 7.

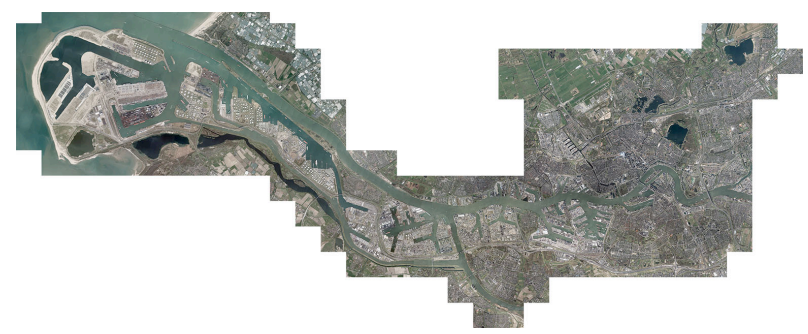

Figure 9. The orthophoto of Rotterdam (RDAM lufo2014.ecw)

\subsection{Use Case 2: The Roman City of Augusta Raurica}

The second use case is a digital reconstruction of the historical Roman City of Augusta Raurica, located some 10 kilometres east of today's city of Basel in Switzerland. Augusta Raurica was one of the largest Roman cities north of the Alps with estimated $15^{\prime} 000$ inhabitants in the $1^{\text {st }}$ and $2^{\text {nd }}$ century AD. The digital reconstruction of the historical city and its continual evolution is part of a long lasting research cooperation between the archaeological team of Augusta Raurica and the Institute of Geomatics Engineering of the University of Applied Sciences and Arts Northwestern Switzerland (FHNW).

The new digital model of Augusta Raurica is the result of a procedural reconstruction based on an accurate excavation GIS database and on the latest scientific findings by the archaeological team. These findings were translated into rule sets for ESRI's CityEngine, which was subsequently used for generating a multi-resolution digital reconstruction of the entire city. This multi-resolution modelling approach allows the new digital model to be used for a broad range of applications. The model with a lower LOD, for example, was used for creating one of the largest bronze models of a historic city (Figures 10, 11) (Schaub, 2014). The high-resolution model featured in this paper, provides the basis for the upcoming «AR App Augusta Raurica», a location-based visitors guide incorporating state-ofthe art augmented reality features. In order to allow for the interactive exploitation of the large and complex 3D model on a standard mobile device, we used the proposed image provisioning approach.

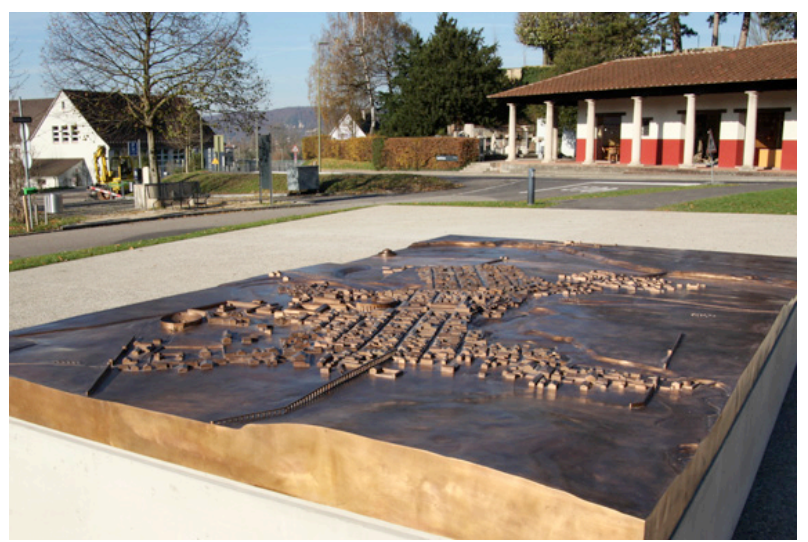

Figure 10. The bronze model of Augusta Raurica, which is on display outside the Roman museum in Augst (Schaub, 2014) 


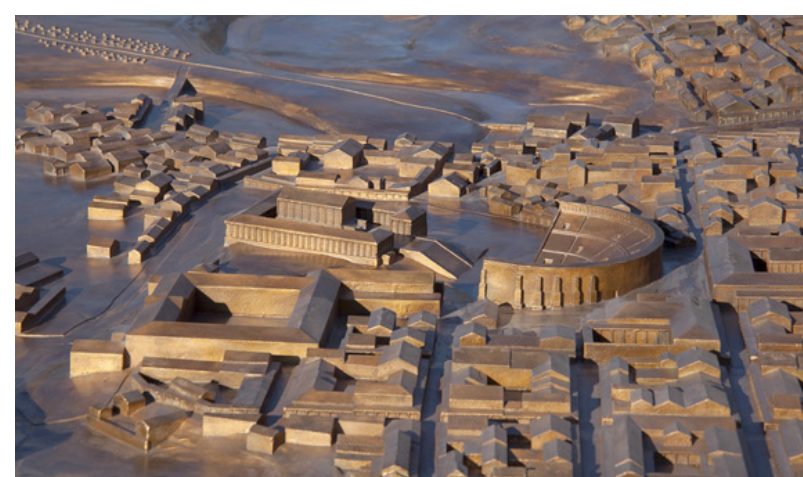

Figure 11. Detail view of the bronze model of Augusta Raurica

The high-resolution 3D model of Augusta Raurica covers an area of approx. $2 \mathrm{~km} \mathrm{x} 2.5 \mathrm{~km}$ and includes over 4000 geospatial objects (such as buildings, roads, vegetation features and the terrain) at three levels of detail. This leads to a geometry dataset of approx. $172 \mathrm{MB}$ and textures in the order of $350 \mathrm{MB}$ (compressed). The rendering is shown in Figures 12 and 13.

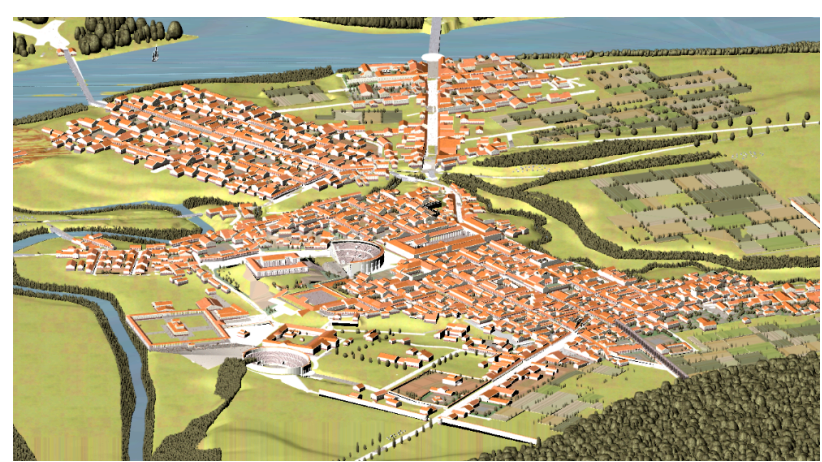

Figure 12: Overview of the rendered Augusta Raurica

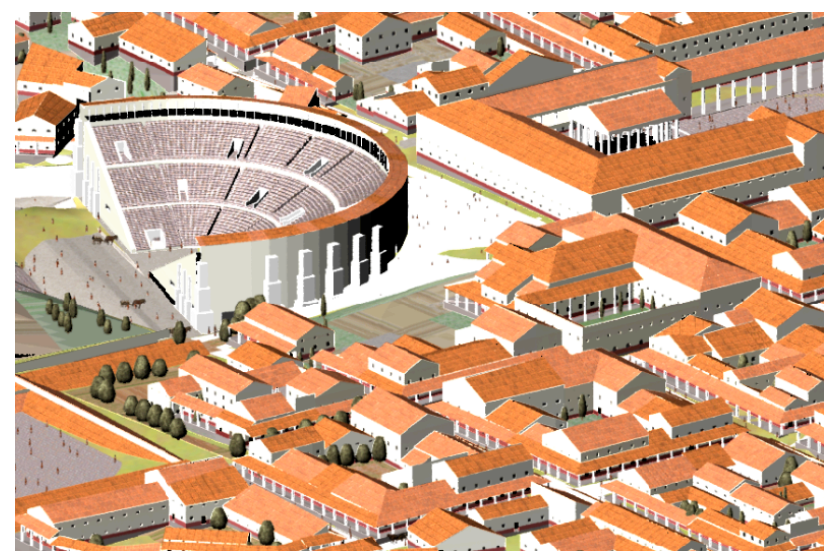

Figure 13: Detail view of the rendered Augusta Raurica

\section{FIRST RESULTS OFFERED BY IMAGE-BASED- PROVISIONING}

For the Augusta Raurica scene, color map tiles (Figure 14) and normal map tiles (Figure 15) are rendered. These two image types are combined on the fly with a simple lighting equation (Figure 16). The lighting is done using the Lambert's cosine law (Lambert, 1760). First, a light direction 1 is defined and then the normal $\mathrm{n}$ is decoded from the normal map. For each pixel, the normalized dot product of 1 and $n$ is multiplied with the $r-$, g- and b-component of the corresponding pixel from the colormap. This allows for dynamic lighting as the light direction may change over time - for example simulating the sun position. This lighting also works without WebGL, since pixels can also be accessed using the canvas API.

In addition to the color map and normal map tiles a color id map is also generated to allow identification and highlighting of buildings (Figure 17). Every color represents an id, supporting a total of $2^{32}$ different ids in an 8-bit per channel RGBA image.

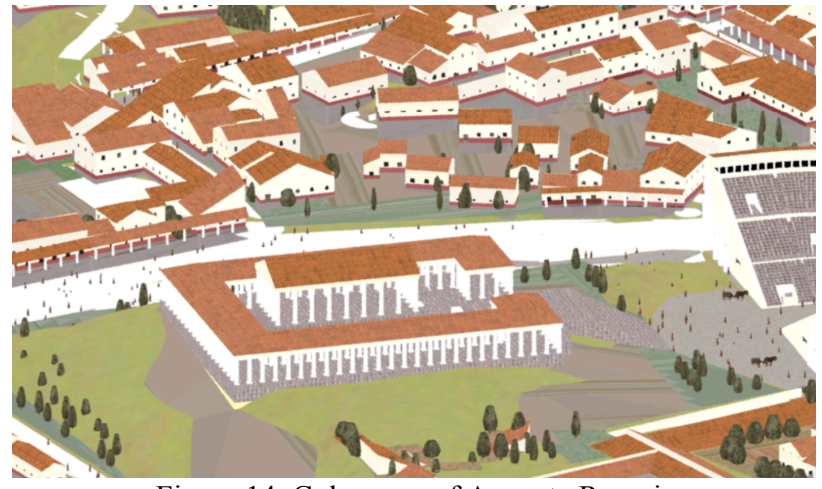

Figure 14. Color map of Augusta Raurcia

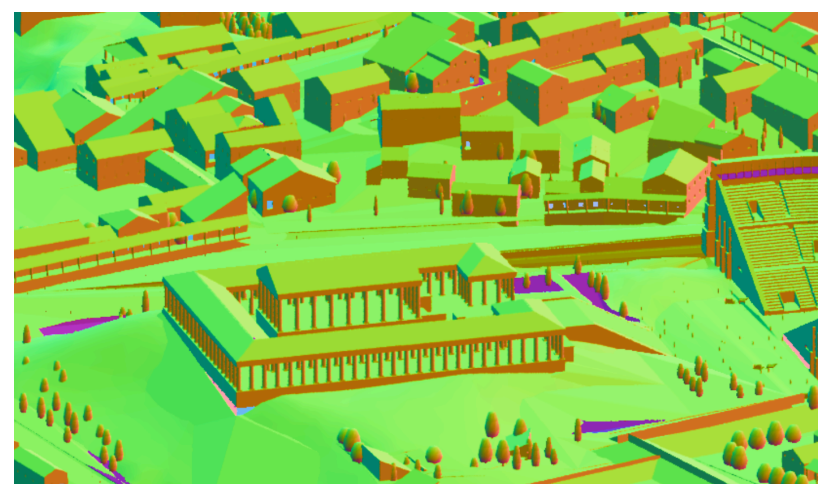

Figure 15. Normal map of Augusta Raurcia

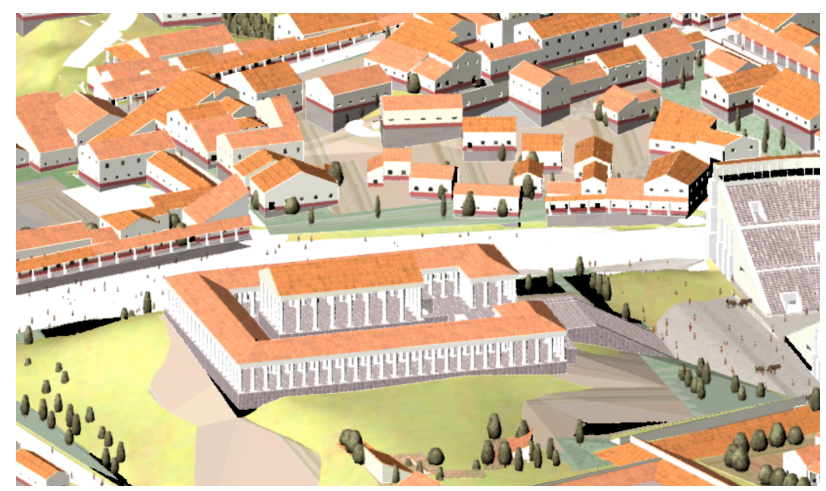

Figure 16. Color and normal map combined with a lighting equation 


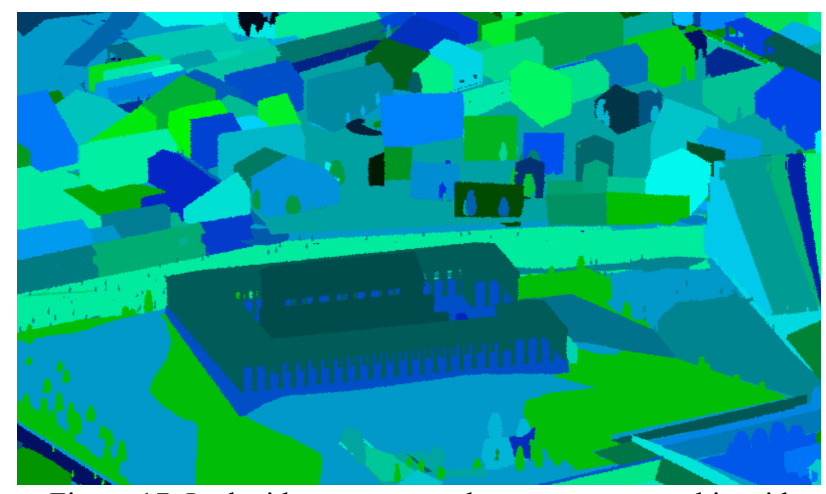

Figure 17. In the id map every color represents an object id

\section{OUTLOOK AND CONCLUSION}

In this paper we introduced a cloud-based approach for 3D image provisioning supporting large and complex 3D city models. The 3D map of Rotterdam and the historic roman city of Augusta Raurica. The city model of Rotterdam has been created from a very large CityGML model with textures and Augusta Raurica has been generated in CityEngine using a procedural modeling approach. The Augusta Raurica case showed that screen space rendering techniques can be used on the fly to create dynamic lighting, even if WebGL is not used. The scenes can be rendered on common mobile and desktop web browsers.

The current state of the view extension of the OGC 3D Portrayal specification has proven to be suitable for the visualisation of different types of city models.

In future, more lighting models will be implemented, such as screen-space ambient occlusion (Kajalin, 2009). In that case the depth map would be used to retrieve the object positions. Another planned feature will be the support of multiple layers. Layers will be combined by using the depth maps of each layer in order to determine which pixel is in front and which in the back. This will also allow to combine scenes with point clouds, as proposed by Nebiker et al., 2010.

Once the OGC 3D Portrayal specification is finalized the 3 dmaps.ch server will be adapted to ensure full compatibility.

\section{REFERENCES}

Barringer R. and Akenine-Möller T., 2014. Dynamic ray stream traversal. ACM Trans. Graph. 33, 4, Article 151 (July 2014), 9 pages.

Christen M., 2005. Implementing Ray Tracing on the GPU. In Wolfgang Engel, editor, ShaderX4 - Advanced Rendering Techniques, pages 413-424. Charles River Media

Christen, M., Nebiker, S. and Loesch, B., 2012. Web-Based Large-Scale 3D-Geovisualisation Using WebGL. International Journal of 3-D Information Modeling, 1(3), pp.16-25.

Christen M., Hürbi K., Nebiker S., 2014. OpenWebGlobe: 3DVisualisierung und Caching von globalen Stadtmodellen aus OpenStreetMap mittels Cloud-basiertem Framework, DGPF Tagungsband 2014, Hamburg

Evans, A. et al., 2014. 3D graphics on the web: A survey. Computers \& Graphics, 41(0), pp.43-61.

Flask, 2015. Flask Documentation / User's Guide, available online http://flask.poco.org/docs/0.10/, accessed April 23, 2015.
Haala, N. and Kada, M., 2010. An update on automatic 3D building reconstruction. ISPRS Journal of Photogrammetry and Remote Sensing, 65(6), pp.570-580.

Haegler, S., Müller, P. \& Van Gool, L., 2009. Procedural Modeling for Digital Cultural Heritage. EURASIP Journal on Image and Video Processing, 2009(852392), pp.1-11.

Hildebrandt D., Klimke J., Hagedorn B., and Döllner J., 2011. Service-oriented interactive 3D visualization of massive 3D city models on thin clients. In Proceedings of the 2nd International Conference on Computing for Geospatial Research \& Applications (COM.Geo '11). ACM, New York, NY, USA

Kajalin V., 2009. Screen-Space Ambient Occlusion. In Wolfgang Engel, editor, ShaderX7 - Advanced Rendering Techniques, pages 413-424. Charles River Media

Klimke J., Hagedorn B., and Döllner J., 2014. Scalable MultiPlatform Distribution of Spatial 3D Contents. Int. J. 3D Inf. Model. 3, 3 (July 2014), 35-49.

Lambert, J. H., 1760. Photometria, sive de Mensura et Gradibus Luminis, Colorum et Umbrae, Augsburg.

Lerner M.R., 2014. At the forge: flask. July 2014 Issue of Linux Journal, Belltown Media, Houston, TX.

MongoDB, 2015. The MongoDB 3.0 Manual, available online http://docs.mongodb.org/manual/, accessed April 20, 2015.

Musialski, P. et al., 2013. A survey of urban reconstruction. Computer Graphics Forum, 32(6), pp.146-177.

Nebiker S., Bleisch S., Christen M., 2010. Rich point clouds in virtual globes - A new paradigm in city modeling?, Computers, Environment and Urban Systems, Volume 34, Issue 6, November 2010, Pages 508-517, ISSN 0198-9715, http://dx.doi.org/10.1016/j.compenvurbsys.2010.05.002.

Open Geospatial Consortium (OGC), 2015. OGC seeks public comment on candidate 3D Portrayal Service Standard, OGC Press Release, last accessed: April 19, 2015 http://www.opengeospatial.org/pressroom/pressreleases/2165

Renderman, 2015. Pixar Renderman Pro Server, available online http://renderman.pixar.com, accessed April 20, 2015.

Rodríguez M. B., Agus M., Marton F., and Gobbetti E., 2014. HuMoRS: huge models mobile rendering system. In Proceedings of the Nineteenth International ACM Conference on 3D Web Technologies (Web3D '14). ACM, New York, NY, USA.

Saito T. and Takahashi T., 1990. Comprehensible rendering of 3-D shapes. In Proceedings of the 17th annual conference on Computer graphics and interactive techniques (SIGGRAPH '90). ACM, New York, NY, USA, 197-206.

Schaub, M., 2014. Das Bronzemodell von Augusta Raurica: vom Stadtplan zum Stadtmodell. Augusta Raurica Magazin, 2014(1), 7-9. 